\title{
Bilateral Multifocal Chorioretinitis and Optic Neuritis due to Epstein-Barr Virus: A Case Report
}

\author{
Vasileios G. Peponis Irini P. Chatziralli \\ Efstratios A. Parikakis Niki Chaira Michail C. Katzakis \\ Panagiotis G. Mitropoulos \\ Second Eye Clinic, Specialized Eye Hospital 'Ophthalmiatreion Athinon', \\ Athens, Greece
}

\section{Key Words}

Epstein-Barr virus · Retina $\cdot$ Chorioretinitis · Optic neuritis

\begin{abstract}
Epstein-Barr virus (EBV) is a DNA virus that mainly causes infectious mononucleosis. Ocular manifestations are rare and typically mild. Only a few cases of EBV involving the retina or the optic nerve have been reported. Herein, we report the case of a 67-year-old man with bilateral chorioretinitis and optic neuritis due to EBV. The patient had no previous ocular history and presented with decreased vision in both eyes. His past medical history included EBV encephalopathy, which was confirmed serologically, a few months before.

Ophthalmological examination revealed bilateral chorioretinitis and optic neuritis, confirmed by fluorescein angiography as well as electrophysiological tests (visual evoked potentials and electroretinogram). It is very important to include EBV in the differential diagnosis of chorioretinal atrophic lesions. Clinicians should be aware of ocular manifestations of EBV, in order to suggest ophthalmological examination and start treatment promptly before irreversible damage to the optic nerve or retina occurs.
\end{abstract}

\section{Introduction}

Epstein-Barr virus (EBV) is a DNA virus of the herpes family and the main cause of infectious mononucleosis (IM) [1]. It has also been associated with Burkitt lymphoma, nasopharyngeal carcinoma, thymic carcinoma, rheumatoid arthritis, Sjögren's syndrome and post-transplant lymphoproliferative disorders [1,2]. Primary infection 
with EBV often occurs in childhood or adolescence and makes the patient a virus carrier, a condition that lasts for life [1].

Ocular manifestations of EBV are rare and typically mild [1]. These include dacryoadenitis, exophthalmos, ophthalmoplegia, cranial nerve palsies, conjunctivitis, keratitis, iritis, optic neuritis, optic disc swelling, uveitis as well as retinal or choroidal involvement [1, 2-20]. Herein, we report the case of bilateral chorioretinitis and optic neuritis in a 67-year-old patient with a history of EBV encephalopathy.

\section{Case Report}

A 67-year-old man with no previous ocular history presented with a 2-month history of decreased vision in both eyes. The patient underwent a thorough ophthalmological examination, including visual acuity (VA) measurement by means of Snellen charts, slit-lamp examination, intraocular pressure (IOP) measurement and dilated fundoscopy. At presentation, his best corrected VA (BCVA) was 6/60 in the right eye and hand movement in the left eye. The anterior segment was totally normal and IOP (15 and $16 \mathrm{~mm} \mathrm{Hg}$ in the right and left eye, respectively) was normal in both eyes as well.

Dilated fundoscopy revealed multiple, well-demarcated gray to white areas of retinal atrophy predominantly involving the periphery in the right eye and the macula in the left eye. Of note, all lesions were on the pathway of retinal vessels. Fluorescein angiography (FA) was performed, showing window defect of the dye in the area of the lesions in late phase (fig. 1). Due to suspicion of optic nerve involvement, the patient was referred for an electrophysiology examination. Flash visual evoked potentials ( $\underline{\text { fig. 2 }}$ ) showed low amplitude in both eyes (6.7 and $5.3 \mu \mathrm{V}$ in the right and the left eye, respectively) and $\mathrm{P}_{100}$ peak time latency in both eyes (149.8 and $146.8 \mathrm{~ms}$ in the right and the left eye, respectively), revealing retrobulbar optic neuritis. An electroretinogram also showed decreased retinal response (ig, 3 ).

As far as his medical history was concerned, the patient had no diabetes mellitus, no hypertension nor any other vascular disease. Eight months earlier, he had been admitted to a general hospital due to fever and skin lesions over his whole body. There was no history of travel in tropical countries, sexual contact, animal exposure or drug abuse; the patient drank occasionally. Written informed consent was obtained by the patient for the publication of this case report.

One day following admission, the patient presented acute confusional state. A cranial computed tomography scan showed generalized brain swelling. The cerebrospinal fluid (CSF) had $850 \mathrm{cells} / \mu \mathrm{l}$ ( $80 \%$ mononuclear cells), a glucose level of $58 \mathrm{mg} / \mathrm{dl}$ (blood glucose of $133 \mathrm{mg} / \mathrm{dl}$ ), a protein level of $127 \mathrm{mg} / \mathrm{dl}$ and an LDH level of $166 \mathrm{U} /$ liter. A complete blood count showed a hematocrit of 35.3\%, a white blood cell (WBC) count of 11,000 cells $/ \mu$ l (83.1\% neutrophils, $10.1 \%$ lymphocytes and $6.7 \%$ monocytes) and a platelet count of 136,000 cells/ $\mu$ l. Blood chemistries, including thyroid testing and transaminases were normal. Blood and CSF bacterial and fungal cultures were negative. The CSF cryptococcal antigen and polymerase chain reaction (PCR) for Mycobacterium tuberculosis were negative. VDRL, FTA-Abs and Treponema pallidum EIA were negative. Real-time quantitative PCR of the CSF was negative for herpes simplex virus, varicella-zoster virus, cytomegalovirus, human herpes virus- 6 and -8, human T-lymphotropic virus and Enterovirus, but was positive for EBV. Interestingly, EBV was not found in blood specimens.

He was treated with ceftriaxone, ampicillin and acyclovir as well as mannitol and dexamethasone for brain swelling. Magnetic resonance imaging was performed due to pyramid signs (Babinski bilaterally) and revealed high-signal intensity and brain swelling, without sings of encephalomyelitis. Therefore, the diagnosis of EBV encephalopathy was confirmed. The patient gradually improved and CSF showed normal levels of WBC (16 cells/ $\mu \mathrm{l})$, glucose $(48 \mathrm{mg} / \mathrm{dl})$ and protein $(59 \mathrm{mg} / \mathrm{dl})$, and PCR was negative for EBV. The patient was discharged home after 2 months of hospitalization and was seen again 3 and 6 months after discharge with nearly complete recovery. Two months after the initial ophthalmologic examination, the patient presented for a follow-up, without having received any treatment. His BCVA had improved to $6 / 18$ in the right eye and counting fingers in the left eye. Dilated fundoscopy revealed the same retinal condition, with the presence of multifocal retinal pigment epithelium atrophy lesions, confirmed by a new FA. 


\section{Discussion}

The reported ocular manifestations of systemic EBV infection are varied and include all segments of the eye [1]. EBV infection should be considered in the differential diagnosis of any atypical ocular inflammatory process [1]. In this case report, we describe a 67-year-old patient with bilateral multiple chorioretinitis and optic neuritis, who presented about 8 months after EBV infection.

Cases of EBV involving the retina are very rare. Kelly et al. [17], Raymond et al. [15] and Kim et al. [2] reported presumed cases of chorioretinitis associated with acute EBV, although all 3 patients were serologically positive for Toxoplasma as well. Similarly, Tiedeman et al. [16] described an association between EBV and multifocal choroiditis. Kramer et al. [18] reported a presumed case of EBV without any laboratory evidence, contrary to Spaide et al. [19] and Bhende et al. [20], who presented serological evidence for their cases of multifocal choroiditis. In our case, the patient had a history of EBV encephalitis, confirmed by serological CSF testing, although blood specimens were normal. Interestingly enough, EBV can cause meningitis, encephalitis, cranial neuropathy, radiculitis and poly- or mononeuritis with or without IM [13]. Our patient did not have previous IM, but PCR in CSF was positive for EBV.

Optic neuritis following EBV infection is also very rare. There have only been 12 cases in the literature [3-14]. Most patients developed optic neuritis between 2 and 12 weeks after IM, and the involvement is limited to the retrobulbar portion $[4-7,10]$. Only a few cases have been reported without IM, which presented with neurological abnormalities and optic neuritis [11-13]. Noticeably, bilateral optic neuritis was described in 4 patients $[5,6,11,13]$. In our case, the onset of neuritis, suspected because of decreased VA, began about 6-8 months after EBV infection, when the patient had totally recovered according to the neurological symptomatology. To our knowledge, this is the fifth case in the literature presenting with bilateral optic neuritis.

The treatment of EBV-associated ocular disease is not well established due to the small number of documented cases [1]. If active inflammation is noted, topical prednisolone acetate 1 drop qid or every 2 hours is recommended [1]. Treatment of IM is usually supportive, as the disease is considered to be self-limited [1]. Systemic acyclovir has been shown effective to inhibit EBV and has been used mainly for immunocompromised patients $[1,2]$. In our case, the patient was given no treatment for the ocular disease, as there was no active inflammation and the previous EBV infection was treated successfully in the past and had a negative PCR.

In conclusion, we report a case of bilateral multifocal chorioretinitis and optic neuritis following EBV encephalopathy. It is very important to include EBV in the differential diagnosis of chorioretinal atrophic lesions. Clinicians should be aware of ocular manifestations of EBV, so as to suggest ophthalmological examination promptly and start treatment before irreversible damage to the optic nerve or retina occurs. 


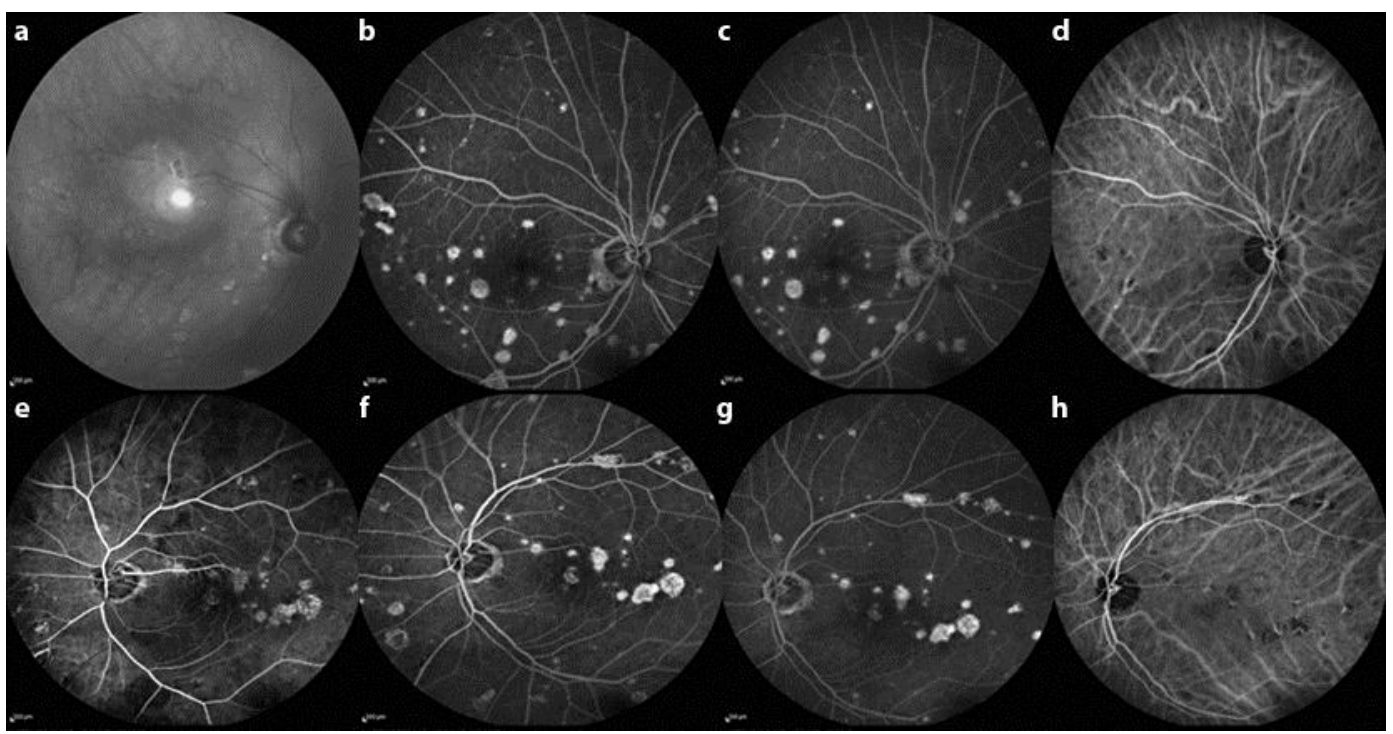

Fig. 1. Red-free (a), fluorescein (b, c) and indocyanine green (d) angiograms in the right eye showing multifocal atrophic lesions of the posterior pole, close to retinal and choroidal vessels. Fluorescein (eg) and indocyanine green (h) angiograms showing multifocal chorioretinitis of the posterior pole, affecting the macula as well.
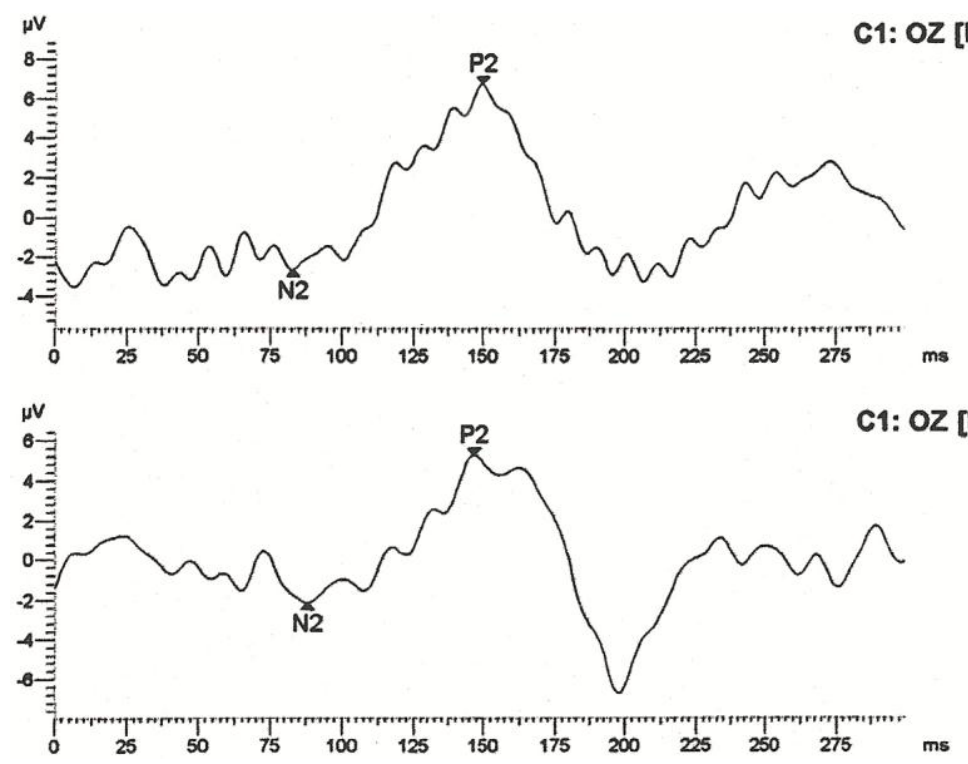

Fig. 2. Flash visual evoked potentials showed low amplitude and peak time latency of P100. 

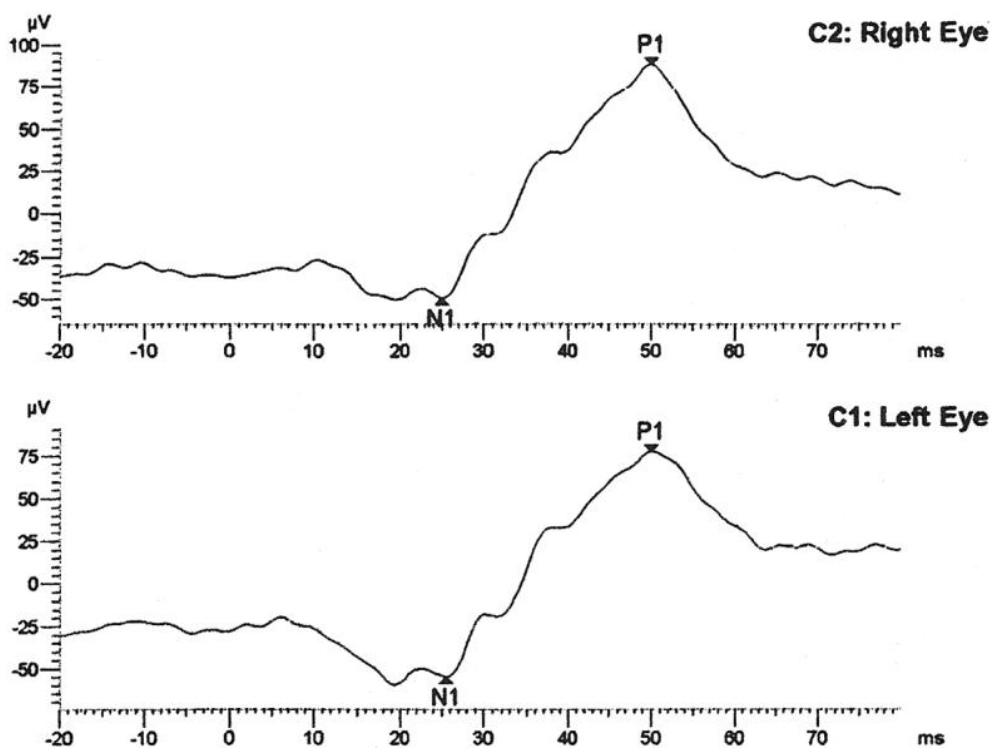

Fig. 3. Electroretinogram showing decreased retinal response.

\section{References}

1 Matoba AY: Ocular disease associated with Epstein-Barr virus infection. Surv Ophthalmol 1990;35:145150.

2 Kim S, Barañano D, Grossniklaus H, Martin D: Epstein-Barr infection of the retina: case report and review of the literature. Retin Cases Brief Rep 2011;5:1-5.

-3 Bonynge TW, Von Hagen KO: Severe optic neuritis in infectious mononucleosis; report of a case. J Am Med Assoc 1952;148:933-934.

-4 Shechter FR, Lipsius EI, Rasansky HN: Retrobulbar neuritis; a complication of infectious mononucleosis. AMA Am J Dis Child 1955;89:58-61.

5 Williamson PM: Neuromyelitis optica following infectious mononucleosis. Proc Aust Assoc Neurol 1975;12:153-155.

6 Pickens S, Sangster G: Retrobulbar neuritis and infectious mononucleosis. Br Med J 1975;4:729.

7 George JL, Roche G, Reny A, Dureux JB: A case of retrobulbar neuritis. A manifestation in infectious mononucleosis. Rev Otoneuroophtalmol 1981;53:411-419.

-8 Jones J, Gardner W, Newman T: Severe optic neuritis in infectious mononucleosis. Ann Emerg Med 1988;17:361-364.

-9 Purvin V, Herr GJ, De Myer W: Chiasmal neuritis as a complication of Epstein-Barr virus infection. Arch Neurol 1988;45:458-460.

10 Anderson MD, Kennedy CA, Lewis AW, Christensen GR: Retrobulbar neuritis complicating acute EpsteinBarr virus infection. Clin Infect Dis 1994;18:799-801.

11 Corssmit EP, Leverstein-van Hall MA, Portegies P, Bakker P: Severe neurological complications in association with Epstein-Barr virus infection. J Neurovirol 1997;3:460-464.

12 Majid A, Galetta SL, Sweeney CJ, Robinson C, Mahalingam R, Smith J, Forghani B, Gilden DH: Epstein-Barr virus myeloradiculitis and encephalomyeloradiculitis. Brain 2002;125:159-165.

13 Phowthongkum P, Phantumchinda K, Jutivorakool K, Suankratay C: Basal ganglia and brainstem encephalitis, optic neuritis, and radiculomyelitis in Epstein-Barr virus infection. J Infect 2007;54:e141e144.

14 An JY, Yoon B, Kim JS, Song IU, Lee KS, Kim YI: Guillain-Barré syndrome with optic neuritis and a focal lesion in the central white matter following Epstein-Barr virus infection. Intern Med 2008;47:15391542. 
15 Raymond LA, Wilson CA, Linnemann CC Jr, Ward MA, Bernstein DI, Love DC: Punctate outer retinitis in acute Epstein-Barr virus infection. Am J Ophthalmol 1987;104:424-426.

16 Tiedeman J: Epstein-Barr viral antibodies in multifocal choroiditis and panuveitis. Am J Ophthalmol 1987;103:659-663.

17 Kelly SP, Rosenthal AR, Nicholson KG, Woodward C: Retinochoroiditis in acute Epstein-Barr virus infection. Br J Ophthalmol 1989;73:1002-1003.

18 Kramer S, Brummer C, Zierhut M: Epstein-Barr virus associated acute retinal necrosis. Br J Ophthalmol 2001;85:114.

19 Spaide RF, Sugin S, Yannuzzi LA, DeRosa JT: Epstein-Barr virus antibodies in multifocal choroiditis and panuveitis. Am J Ophthalmol 1991;112:410-413.

20 Bhende PS, Biswas J, Madhavan HN: Serological evidence for active Epstein-Barr virus infection in multifocal choroiditis. Indian J Pathol Microbiol 1997;40:129-131. 